\title{
Ki-67 Labeling Index in Primary Invasive Breast Cancer from Sudanese Patients: A Pilot Study
}

\author{
Khalid Dafaallah Awadelkarim, ${ }^{1}$ Renato Mariani-Costantini, ${ }^{2}$ \\ Ihsan Osman, ${ }^{3}$ and Massimo Costanzo Barberis ${ }^{4}$ \\ ${ }^{1}$ Department of Molecular Biology, National Cancer Institute (NCI-UG), University of Gezira, P.O. Box 20, \\ Hospital Street, Wad Medani, Sudan \\ ${ }^{2}$ Department of Medicine, Dentistry and Biotechnology, "G. d'Annunzio" University and Unit of General Pathology, \\ Aging Research Center (CeSI), “G. d'Annunzio” University Foundation, via Colle dell'Ara, 66013 Chieti, Italy \\ ${ }^{3}$ Department of Histopathology \& Cytopathology, Radiation \& Isotope Centre Khartoum (RICK), Algaser Street, \\ P.O. Box 846, Khartoum, Sudan \\ ${ }^{4}$ Division of Pathology, European School of Oncology (IEO), via Ripamonti 435, 20141 Milan, Italy
}

Correspondence should be addressed to Khalid Dafaallah Awadelkarim, awadelkarim@gmail.com

Received 6 November 2012; Accepted 22 November 2012

Academic Editors: L. Colomo, K. Nakanishi, and M. A. Sughayer

Copyright ( $\odot 2012$ Khalid Dafaallah Awadelkarim et al. This is an open access article distributed under the Creative Commons Attribution License, which permits unrestricted use, distribution, and reproduction in any medium, provided the original work is properly cited.

Ki-67 labeling index has been linked to patient outcome in breast cancer patients. However, very few published reports have examined Ki-67 labeling index in African breast cancer patients. Sixty-two Sudanese breast cancer patients with primary invasive tumors were immunostained for Ki-67, ER, PR, Her-2/neu, CK5/6, and CK17. Ki-67 labeling index ranged from 0\% to 50\%, with a median of $5 \%$ (interquartile range $0-10$ ). Low Ki-67 labeling index (immunostaining < 10\%) was detected in 43/62 (69.4\%) with a median of 0 (interquartile range 0-5), whereas high Ki-67 labeling index (immunostaining $\geq 10 \%$ ) was revealed in 19/62 (30.6\%) with a median of 20 (interquartile range 12-26). Ki-67 labeling index was significantly associated with tumor grade $(P=0.022$, Mann-Whitney $U$ Test). There were no significant group differences between Ki-67 labeling index and $\mathrm{ER}(P=0.43), \mathrm{PR}(P=0.7)$, Her-2/neu $(P=0.45)$, CK5/6 $(P=0.29)$, CK17 $(P=0.55)$, pathologic stage $(P=0.4)$, tumor histology $(P=0.99)$, breast cancer subtypes $(P=0.47)$, tumor size $(P=0.16)$, and age at diagnosis $(P=0.6)$. These results suggested that Ki-67 labeling index correlates with tumor differentiation and not with the tumor size or any other tested marker in Sudanese breast cancers. Thus, Ki-67 labeling index could be considered as a reliable measure of tumor proliferative fraction in Sudan.

\section{Introduction}

The prognosis of breast cancer, a complex and heterogeneous disease $[1,2]$, depends on several clinical/pathological and biological features such as lymph node (LN) status, tumor size and tumor grade [3-5]; presence or absence of hormone receptors (estrogen (ER) and progesterone $(\mathrm{PR})$ ) and other biomarkers, with particular regard to the expression levels of the human epidermal receptor type 2 (Her-2/neu) [6-8]. Data on prognostic biomarkers for breast cancer in indigenous African patients are very scarce due to a number of economic, logistic, and technical reasons $[9,10]$. Previously we reported that a breast cancer case series from Khartoum,
Central Sudan, was comparable to a breast cancer case series from Milan, Northern Italy, in combined hormone receptors status and breast cancer subtypes [10]. However, relative to the Italian patients, the Sudanese patients were younger and their tumors were larger, of higher grade and more advanced in stage [10]. Furthermore, in this Sudanese series the basal cytokeratins (basal CKs) were expressed in a fraction of cases comparable to those reported for East and West African breast cancer case series, and the combined basal CK positive status was associated with higher grade and inversely correlated with ER, PR, and combined hormone receptor status (ER and/or PR) [11]. Of note, the combined basal CK positive status was not linked to age at diagnosis and 
tumor size, which may represent a special feature of basal-like breast cancer in Sudan [11].

$\mathrm{Ki}-67$ is a nuclear protein with varied expression intensity throughout the cell cycle: levels of Ki-67 are low during G1- and early S-phase and progressively increase to reach a maximum during mitosis [12]. The Ki-67 labeling index (LI), assessed using the monoclonal antibody MIB-1 [13], is an excellent marker for determining the growth fraction of tumor cell populations (growth fraction) and hence the biological aggressiveness because apoptosis, or programmed cell death, has been consistently reported to be positively correlated with Ki-67 [12]. An index based on the Ki-67 apoptosis ratio has been developed to approximate the contribution that these two factors may make to tumor growth [12]. While it is clear that this cannot accurately reflect growth dynamics, it may have utility as an early marker of response to treatment in primary therapy of breast cancer [12]. The Ki-67 LI, a reliable measure of tumor proliferative fraction, has extensively been studied and shown to have independent prognostic significance in several large clinical trials of adjuvant endocrine therapy [14-16]. However, it is not currently accepted as a standard biomarker in breast cancer, because of the need for standardization and detailed guidelines [17].

In Sudan, as well as elsewhere in Africa, data concerning the associations between $\mathrm{Ki}-67 \mathrm{LI}$ and the pathologi$\mathrm{cal} /$ clinical parameters of breast cancer are lacking. The current pilot study intended to investigate the association(s) between the Ki-67 LI and individual, pathological, and clinical and immunohistochemical characteristics in pilot study of 62 patients diagnosed with primary invasive breast cancer at RICK, Sudan.

\section{Materials and Methods}

2.1. Patients. The study is based on a series of 62 Sudanese breast cancer patients with primary invasive tumors diagnosed between 2004 and 2005 at the Department of Histopathology \& Cytopathology of the Radiation and Isotope Center Khartoum (RICK), Khartoum, Sudan. Age and tumor size were recorded only in $40(64.5 \%)$ and $53(85.5 \%)$ of the 62 patients, respectively (Table 1). Breast cancers were classified and staged according to the World Health Organization classification [3] and American Joint Committee on Cancer [5]. Histological grading was performed using the Nottingham Combined Histologic Grade (NCHG) system [4]. Unfortunately, most patients presented with advanced disease and were lost to followup, as it frequently occurs in developing countries [18-20]. Lack of proper data on lymph node status (Table 1), metastatic disease status, and followup precluded correlations with disease stage and prognosis $[10,11]$. According to data from the Sudan Federal Ministry of Health, $78 \%$ of the Sudanese breast cancer patients have stage III or IV disease [21, 22].

2.2. Immunohistochemistry. Whole consecutive sections were immunostained for Ki-67 (clone MIB-1, Dako), ER (clone 1D5, Dako), PR (clone PgR 636, Dako), Her-2/neu (polyclonal, Dako), CK5/6 (clone D5/16 B4, Dako), CK17 (clone
TABLE 1: Individual, pathological, and clinical characteristics of the studied patients.

\begin{tabular}{lc}
\hline Age $(\text { years })^{\natural}$ & \\
Mean \pm SD & $56.4 \pm 13$ \\
Range & $35-80$ \\
Tumor size $(\mathrm{cm})^{\#}$ & \\
Mean \pm SD & $4.4 \pm 2.1$ \\
Range & $1-10$ \\
\hline \multicolumn{2}{l}{ Number $(\%)$} \\
\hline Histology & \\
IDC* & $54(87.1 \%)$ \\
ILC & $3(4.8 \%)$ \\
Mucinous* & $4(6.5 \%)$ \\
Medullary & $1(1.6 \%)$ \\
Grade & \\
2 & $20(32.3 \%)$ \\
3 & $42(67.7 \%)$ \\
Pathologic stage (pT) & \\
T1 & $7(11.3 \%)$ \\
T2 & $29(46.8 \%)$ \\
T3 & $14(22.6 \%)$ \\
T4 & $3(4.8 \%)$ \\
Tx & $9(14.5 \%)$ \\
L.N Status & \\
Positive & $15(24.2 \%)$ \\
Negative & $1(1.6 \%)$ \\
NX & $46(74.2 \%)$ \\
\hline
\end{tabular}

"Age information was available for 40 patients; ${ }^{\#}$ tumor size data was available for 53 patients; ${ }^{*}$ includes one case with bilateral breast cancer.

E3, Dako), and, as quality controls of antigenic preservation, CK pool (clones AE1-AE3, Dako) and vimentin (clone V9, Dako). Antigen retrieval was done using Water bath Tris/EDTA buffer for ER, PR, CK5/6, and CK17; microwave/citrate buffer for Her-2/neu and Vimentin; and Pronase for CK pool. Slides were immunostained by Dako Autostainer Plus (Dako, Glostrup, Denmark).

IHC results were recorded as percentages of immunostained cells in $\geq 2000$ neoplastic cells. Only nuclear reactivity was taken into account for ER and PR, which were classified as negative, when absent or present in $<5 \%$ of the neoplastic cells or positive, when present in $\geq 5 \%$ of the neoplastic cells. Only intense and complete cell membrane immunoreactivity in $\geq 10 \%$ of the cells was taken as evidence of Her- $2 /$ neu overexpression (score 3+). Patients with borderline Her2/neu status (score 2+) were reassessed by fluorescence in situ hybridization (FISH), as previously described [10]. Basal CKs 5/6 and 17 were regarded as positive when any cytoplasmic and/or cell membrane staining was seen. Breast cancer subtypes were classified as described previously [10].

2.3. Determination of the Ki-67 Labeling Index (LI) MIB-1 Immunostaining. Nuclear reactivity was taken into account for $\mathrm{Ki}-67$, which was recorded as continuous variables, based on the proportion of positive tumor cells $(0 \%-100 \%)$ 
TABle 2: Immunohistochemistry characteristics of the studied patients.

\begin{tabular}{lc}
\hline & Number $(\%)$ \\
\hline ER & \\
Positive & $40(64.5 \%)$ \\
Negative & $22(35.5 \%)$ \\
PR & \\
Positive & $48(77.4 \%)$ \\
Negative & $14(22.6 \%)$ \\
Her-2/neu & \\
Positive & $12(19.4 \%)$ \\
Negative & $50(80.6 \%)$ \\
CK5/6 & \\
Positive & $8(12.9 \%)$ \\
Negative & $50(80.6 \%)$ \\
NA & $4(6.5 \%)$ \\
CK17 & \\
Positive & $20(32.3 \%)$ \\
Negative & $40(64.5 \%)$ \\
NA & $2(3.2 \%)$ \\
Breast Cancer Subtypes & \\
Luminal A & $44(71 \%)$ \\
Luminal B & $6(9.7 \%)$ \\
Her-2/neu+ & $6(9.7 \%)$ \\
Basal-like & $4(6.5 \%)$ \\
Unclassified & $2(3.2 \%)$ \\
\hline
\end{tabular}

NA: not available.

regardless of staining intensity (Ki-67 LI). We presented the values of $\mathrm{Ki}-67 \mathrm{LI}$ as range, median, and interquartile (IQ) range.

Besides evaluating Ki-67 as continuous variable, levels of Ki-67 LI were dichotomized as high Ki-67 LI (immunostaining $\geq 10 \%$ ) and low Ki-67 LI (immunostaining $<10 \%$ ) as an arbitrary choice, as published elsewhere [23]. We did not use the median of the distribution of Ki-67 LI among the studied patients as cutoff value due to the small size of the studied patients $(n=62)$.

2.4. Statistical Analyses. The normal distribution of Ki67LI values was verified by using normal Q-Q plot, with the Blom's method. Nonparametric Mann-Whitney $U$ and Kruskal-Wallis tests were used to investigate group differences between Ki-67 LI and ER, PR, Her-2/neu, CK5/6, CK17, pathologic stage ( $\mathrm{pT})$, tumor histology, breast cancer subtypes, and tumor grade, whereas Spearman rank correlation test was used to investigate the relationship between Ki-67 LI and age at diagnosis and tumor size and to assess the relationships between the different clinical/pathological variables of the studied breast cancer patients.

All cut-off values were determined before the statistical procedures. Significance was set at $<0.05$. All $P$ values were two-tailed.

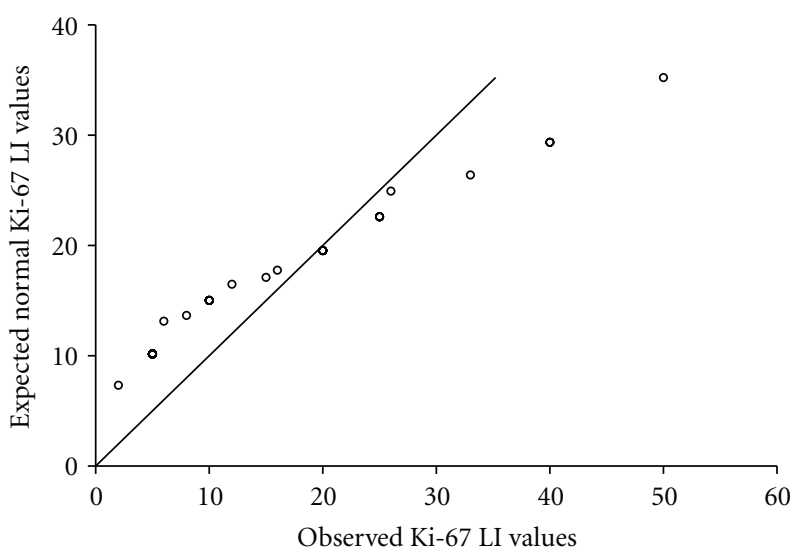

FIGURE 1: The normal distribution of Ki-67 LI values using normal Q-Q plot, with the Blom's method.

Table 3: Ki-67 Labeling Index (LI) "MIB-1 immunostaining" in the studied patients.

\begin{tabular}{lc}
\hline & Number \\
\hline Ki-67 (MIB-1) as continuous variable & 5 \\
Median & $0-10$ \\
Interquartile (IQ) range & $0-50$ \\
Range & \\
Ki-67 (MIB-1) as category variable & $43(69.4 \%)$ \\
Low Ki-67 LI (immunostaining $<10 \%)^{\#}$ & $19(30.6 \%)$ \\
$\quad$ High Ki-67 LI (immunostaining $\geq 10 \%)^{\diamond}$ & \\
\#Median: 0 (interquartile range 0-5). ${ }^{\circ}$ Median: 20 (interquartile range $12-$ \\
26).
\end{tabular}

\section{Results}

The individual, pathological, and clinical characteristics of the studied patients are shown in Table 1. The mean age was $56.4 \pm 13$ years (range: $35-80$ years). The mean tumor size was $4.4 \pm 2.1$ (range: $1-10 \mathrm{~cm}$ ). Overall, the most frequent histotype was invasive ductal carcinoma, which accounted for $54 / 62$ patients $(87.1 \%)$. Other histotypes were invasive lobular $(3 / 62,4.8 \%)$, mucinous $(4 / 62,6.5 \%)$, and medullary $(1 / 62,1.6 \%)$. All tumors were of intermediate and high grade (grade 2: 20/62 (32.3\%); grade 3: $42 / 62(67.7 \%)$ ). The immunohistochemical characteristics of the studied patients were shown in Table 2. ER. PR, Her-2/neu, CK5/6, and CK17 positivity was $64.5 \%$ (40/62), $77.4 \%$ (48/62), $19.4 \%$ (12/62), $12.9 \%(8 / 62)$, and $32.3 \%(20 / 62)$, respectively. The most frequent breast cancer subtype in the studied patients was luminal A $(44 / 62,71 \%)$, followed by luminal B and Her$2 /$ neu $+(6 / 62,9.7 \%$ each $)$, whereas the frequency of basallike subtype was $(4 / 62,6.5 \%)$. There were two patients (3.2\%) with unclassified subtype.

3.1. Ki-67 LI (MIB-1) as Continuous Variable. The normal distribution of Ki-67LI values was shown in Figure 1. The Ki-67 LI ranged from 0 to $50 \%$, with a median of $5 \%$ (IQ range: $0-10$ ) (Table 3). In the studied patients the Ki-67 LI was significantly associated with tumor grade $(P=0.022$, 
TABLE 4: Relationships between the different clinical/pathological variables of the studied breast cancer patients.

\begin{tabular}{|c|c|c|c|c|c|c|c|c|c|}
\hline & Ki-67 LI* & Tumor grade & Tumor size & ER & $\mathrm{PR}$ & HER-2/neu & Age & $\begin{array}{c}\text { Breast cancer } \\
\text { subtypes }\end{array}$ & CK5/6 \\
\hline \multirow{2}{*}{ Tumor grade } & $0.293^{\diamond}$ & & & & & & & & \\
\hline & $0.021^{\#}$ & & & & & & & & \\
\hline \multirow{2}{*}{ Tumor size } & -0.195 & -0.098 & & & & & & & \\
\hline & 0.162 & 0.487 & & & & & & & \\
\hline \multirow{2}{*}{ ER } & 0.102 & 0.079 & 0.181 & & & & & & \\
\hline & 0.431 & 0.541 & 0.195 & & & & & & \\
\hline \multirow{2}{*}{ PR } & 0.050 & 0.373 & 0.083 & 0.567 & & & & & \\
\hline & 0.698 & 0.003 & 0.555 & $<0.001$ & & & & & \\
\hline \multirow{2}{*}{ HER-2/neu } & -0.098 & -0.163 & 0.023 & -0.319 & -.419 & & & & \\
\hline & 0.449 & 0.204 & 0.872 & 0.011 & 0.001 & & & & \\
\hline \multirow{2}{*}{ Age } & -0.083 & 0.198 & -0.077 & 0.064 & 0.037 & -0.112 & & & \\
\hline & 0.612 & 0.220 & 0.657 & 0.693 & 0.823 & 0.492 & & & \\
\hline \multirow{2}{*}{ Breast cancer subtypes } & -0.011 & 0.313 & 0.109 & 0.612 & 0.827 & -.650 & 0.126 & & \\
\hline & 0.931 & 0.013 & 0.436 & $<0.001$ & $<0.001$ & $<0.001$ & 0.437 & & \\
\hline \multirow{2}{*}{ CK5/6 } & -0.139 & -0.160 & 0.095 & -0.341 & -0.265 & -0.066 & 0.070 & -0.229 & \\
\hline & 0.298 & 0.229 & 0.517 & 0.009 & 0.045 & 0.623 & 0.685 & 0.084 & \\
\hline \multirow{2}{*}{ CK17 } & -0.077 & -0.125 & 0.056 & -0.148 & -0.143 & -0.088 & -0.278 & -0.111 & 0.246 \\
\hline & 0.557 & 0.341 & 0.694 & 0.258 & 0.276 & 0.502 & 0.091 & 0.398 & 0.067 \\
\hline
\end{tabular}

${ }^{*}$ Ki-67 LI as continuous variable ${ }^{\diamond}$ Spearman correlation coefficient (Spearman's rho); ${ }^{*} P$ value of Spearman rank correlation test, significant correlations were marked in bold.

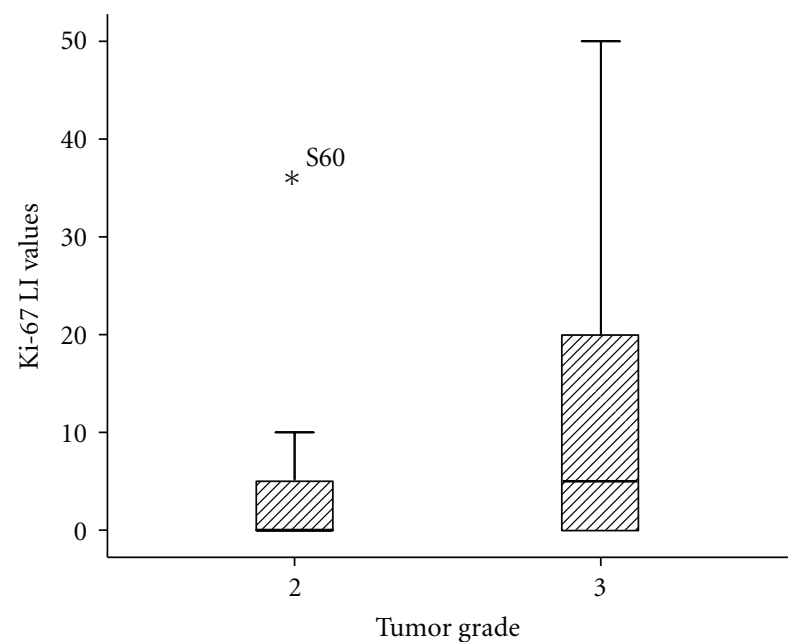

FIGURE 2: Boxplot showing the distribution of Ki-67 LI values in the studied patients according to the tumor grade (Mann-Whitney $U$, $P=0.022$ ).

Mann-Whitney $U$ Test). In fact the median Ki-67 LI for grade 3 tumors (median: 5; IQ range: 0-20) was higher than the median Ki-67 LI for grade 2 tumors (median: 0; IQ range: $0-5)$, (Figure 2). There were no significant group differences between Ki-67 LI and ER $(P=0.43)$ (Figure 3), PR $(P=0.7)$, Her-2/neu $(P=0.45)$, CK5/6 $(P=0.29)$,
CK17 $(P=0.55)$, pathologic stage "pT" $(P=0.4)$, tumor histology $(P=0.99)$, and breast cancer subtypes $(P=0.47)$. Moreover, there was no correlation between Ki-67 LI and tumor size (Spearman's rho $=-0.195, P=0.16$ ) and age at diagnosis (Spearman's rho $=-0.083, P=0.6$ ).

Detailed description of the relationship between the different tested clinical/pathological variables including $\mathrm{Ki}$ 67 in the studied patients is shown in Table 4. Of note, ER was negatively correlated with Her-2/neu (Spearman's rho = $0.319, P=0.011)$, and PR was correlated with tumor grade (Spearman's rho $=0.373, P=0.003$ ).

3.2. Ki-67 LI (MIB-1) as Category Variable. Low Ki-67 LI (immunostaining < 10\%) was detected in 43/62 (69.4\%) with a median of 0 (interquartile range $0-5$ ), whereas, High Ki-67 LI (immunostaining $\geq 10 \%$ ) was revealed in 19/62 $(30.6 \%)$ with a median of 20 (interquartile range 12-26) (Table 3). Similar to Ki-67 LI as continuous variable, the dichotomized Ki-67 LI was not associated with any of the tested variables, except the detected association between $\mathrm{Ki}$ $67 \mathrm{LI}$ and tumor grade $(P=0.019$, Table 5).

\section{Discussion}

Although Ki-67 LI has been linked to the outcome and/or to the clinical and pathological variables of breast cancer patients [14-16], very few published reports have examined Ki-67 LI in African breast cancer patients [24, 25]. Published data on breast cancer from Sudan is lacking, and to our 
TABLE 5: The relationship between tumor grade and dichotomized Ki-67 Labeling Index (LI) using 10\% as cutoff value in the studied patients.

\begin{tabular}{cccc}
\hline & & \multicolumn{2}{c}{ Tumor grade } \\
\hline & & 2 & 3 \\
Ki-67 LI & Low (immunostaining $<10 \%)$ & $18(90 \%)$ & $25(59.5 \%)$ \\
$($ MIB-1) & High (immunostaining $\geq 10 \%)$ & $2(10 \%)$ & $17(40.5 \%)$ \\
& Fisher's Exact Test & \multicolumn{2}{c}{$P=0.019$} \\
\hline
\end{tabular}

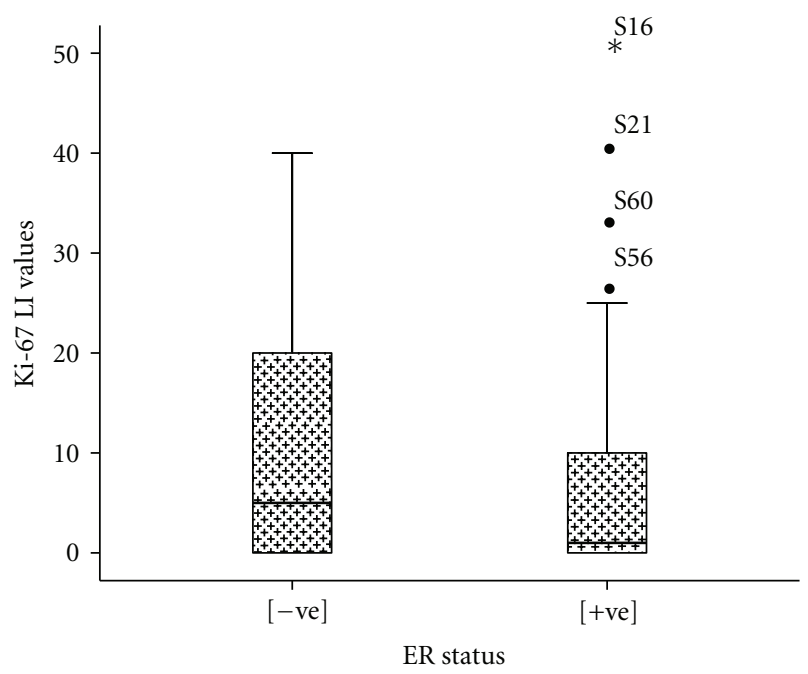

Figure 3: Boxplot showing the distribution of Ki-67 LI values in the studied patients according to the ER status (Mann-Whitney $\mathrm{U}, P=0.43$ ). The median Ki-67 LI of ER-negative tumors was 5 (interquartile range 0-19), whereas the median Ki-67 LI of ERpositive tumors was 1 (interquartile range $0-10$ ).

knowledge no studies had been conducted on Ki-67 LI in breast cancer patients from Sudan.

In this paper we investigated the relationship between Ki-67 labeling index and individual and clinical/pathological characteristics of primary invasive breast cancers from Sudan. The majority of the studied patients were premenopausal young women with high grade and large tumors; this could be attributable to a lower frequency of postmenopausal breast cancer due to population structure and/or age-related biases in seeking cure, as well as to late stage at disease diagnosis $[10,21,22,26]$. This situation is a reflection of the healthcare crisis in sub-Saharan Africa $[9,10,26]$.

In the current study Ki-67 LI was significantly associated with tumor grade $(P=0.022$, Mann-Whitney $U$ Test $)$, and the median of Ki-67 LI of grade 3 tumors was higher than that of grade 2 tumors ( 5 versus 0 , resp.). This is in agreement with the published literature; in fact in most studies histological grade was reported to be linked to Ki-67 [12]. This is to be expected given that mitotic index is one of three components of grade and Ki-67 labeling is commonly used as a complement to grading systems $[12,17]$.

We evaluated Ki-67 as continuous variable and as reliable measure, because it has been shown that models with continuous values provide more information [27]. Also transforming continuous variables, such as the Ki-67 labeling index, into two categories can lead to a loss of power of the biomarker [17]. It is worth mentioning that this was observed when we use the median $\mathrm{Ki}-67 \mathrm{LI}$ among the studied patients as cutoff value in relation to tumor grade (data not shown). However, the association between dichotomized Ki-67 LI and tumor grade persisted when using 10\% as arbitrary cutoff value $(P=0.019$, Table 5$)$. This might indicate that a cutoff value of $10 \%$ is more reliable [23]. However, at the individual level, this might be unrealistic, since it suggests that patients, who have tumors with Ki-67 levels close to the cutoff value but on either side of cutoff value, are very different, whereas in reality they are probably very similar [17].

In the studied patients Ki-67 LI was not linked to any of the other tested variables, that is, ER, PR, Her-2/neu, CK5/6, CK17, tumor histology and breast cancer subtypes, and age at diagnosis. Also, there was no correlation between $\mathrm{Ki}-67 \mathrm{LI}$ and tumor size, either as continuous variable or as categorical variable (pathologic stage "pT"). The lack of association between the tested variables and Ki-67 LI might be due to the small size of the current study. However, the lack of association between Ki-67 LI and ER is intriguing, because higher rates of ER positivity are shown in the least proliferating tumors, and only a few studies contradicted this finding $[12,17]$. In the current study the median of ER negative tumors was higher than the median of ER positive tumors (5 versus 1, Figure 3 ), which did not reach statistical significance, maybe due to the small study size as mentioned previously, but also might represent a special feature of breast cancer in Sudan, mainly due to the presence of many high grade ER-positive tumors, for example, S16, S21, S56, and S60 (Figure 3). Hence high grade may represent a confounding factor for the association of Ki-67 LI and ER-positive breast cancer tumors from Sudan.

\section{Conclusion}

In conclusion, our results suggested that Ki-67 labeling index correlates with tumor differentiation and not with the tumor size or any other tested marker in Sudanese breast cancers. Thus, Ki-67 labeling index could be considered as a reliable measure of tumor proliferative fraction in Sudan.

\section{Conflict of Interests}

The authors declare no conflict of interests.

\section{Acknowledgments}

The authors wish to acknowledge the support of a grant from the Sudanese Federal Ministry of Higher Education and Scientific Research "research project: Molecular Subtypes and Risk Factors of Breast Cancer in Sudan" to KDA. The authors also wish to acknowledge the support of a generous grant from "Fondazione Cassa di Risparmio della Provincia di Chieti", Chieti, Italy, for the development of cooperation in breast cancer pathology between "G. d'Annunzio" University 
of Chieti-Pescara, Italy and the Sudanese National Cancer Institute, University of Gezira, Wad Medani, Sudan.

\section{References}

[1] C. M. Perou, T. Sørile, M. B. Eisen et al., "Molecular portraits of human breast tumours," Nature, vol. 406, no. 6797, pp. 747752,2000

[2] T. Sorliea, C. M. Perou, R. Tibshirani et al., "Gene expression patterns of breast carcinomas distinguish tumor subclasses with clinical implications," Proceedings of the National Academy of Sciences of the United States of America, vol. 98, no. 19, pp. 10869-10874, 2001.

[3] F. A. Tavassoli and P. Devilee, Eds., World Health Organization Classification of Tumours. Pathology and Genetics of Tumours of the Breast and Female Genital Organs, IARC Press, Lyon, France, 2003.

[4] J. F. Simpson, R. Gray, L. G. Dressier et al., "Prognostic value of histologic grade and proliferative activity in axillary nodepositive breast cancer: results from the Eastern Cooperative Oncology Group companion study, EST 4189," Journal of Clinical Oncology, vol. 18, no. 10, pp. 2059-2069, 2000.

[5] F. L. Greene, D. L. Page, I. D. Fleming et al., Eds., AJCC Cancer Staging Manual, Springer, New York, NY, USA, 6th edition, 2002.

[6] W. F. Anderson, B. E. Chen, I. Jatoi, and P. S. Rosenberg, "Effects of estrogen receptor expression and histopathology on annual hazard rates of death from breast cancer," Breast Cancer Research and Treatment, vol. 100, no. 1, pp. 121-126, 2006.

[7] V. J. Bardou, G. Arpino, R. M. Elledge, C. K. Osborne, and G. M. Clark, "Progesterone receptor status significantly improves outcome prediction over estrogen receptor status alone for adjuvant endocrine therapy in two large breast cancer databases," Journal of Clinical Oncology, vol. 21, no. 10, pp. 1973-1979, 2003.

[8] G. Arpino, S. J. Green, D. C. Allred et al., "HER-2 amplification, HER-1 expression, and tamoxifen response in estrogen receptor-positive metastatic breast cancer: a southwest oncology group study," Clinical Cancer Research, vol. 10, no. 17, pp. 5670-5676, 2004.

[9] K. D. Awadelkarim, A. A. Mohamedani, and M. Barberis, "Role of pathology in sub-Saharan Africa: an example from Sudan," Pathology and Laboratory Medicine International, vol. 2, pp. 49-57, 2010

[10] K. D. Awadelkarim, C. Arizzi, E. O. M. Elamin et al., "Pathological, clinical and prognostic characteristics of breast cancer in Central Sudan versus Northern Italy: implications for breast cancer in Africa," Histopathology, vol. 52, no. 4, pp. 445-456, 2008.

[11] K. D. Awadelkarim, C. Arizzi, E. O. Musa et al., "Basal-like phenotype in a breast carcinoma case series from Sudan: prevalence and clinical/pathological correlations," Pathology Research International, vol. 2011, Article ID 806831, 10 pages, 2011.

[12] A. Urruticoechea, I. E. Smith, and M. Dowsett, "Proliferation marker Ki-67 in early breast cancer," Journal of Clinical Oncology, vol. 23, no. 28, pp. 7212-7220, 2005.

[13] D. McCormick, H. Chong, C. Hobbs, C. Datta, and P. A. Hall, "Detection of the Ki-67 antigen in fixed and wax-embedded sections with the monoclonal antibody MIB1," Histopathology, vol. 41, no. 3, pp. 173-178, 2002.

[14] E. De Azambuja, F. Cardoso, G. De Castro et al., "Ki-67 as prognostic marker in early breast cancer: a meta-analysis of published studies involving 12155 patients," British Journal of Cancer, vol. 96, no. 10, pp. 1504-1513, 2007.
[15] G. Viale, M. M. Regan, E. Maiorano et al., "Prognostic and predictive value of centrally reviewed expression of estrogen and progesterone receptors in a randomized trial comparing letrozole and tamoxifen adjuvant therapy for postmenopausal early breast cancer: BIG 1-98," Journal of Clinical Oncology, vol. 25 , no. 25, pp. 3846-3852, 2007.

[16] G. Viale, A. Giobbie-Hurder, M. M. Regan et al., "Prognostic and predictive value of centrally reviewed Ki-67 labeling index in postmenopausal women with endocrine-responsive breast cancer: results from breast international group trial 198 comparing adjuvant tamoxifen with letrozole," Journal of Clinical Oncology, vol. 26, no. 34, pp. 5569-5575, 2008.

[17] E. Luporsi, F. André, F. Spyratos et al., "Ki-67: level of evidence and methodological considerations for its role in the clinical management of breast cancer: analytical and critical review," Breast Cancer Research and Treatment, vol. 132, no. 3, pp. 895$915,2012$.

[18] A. Gondos, H. Brenner, H. Wabinga, and D. M. Parkin, "Cancer survival in Kampala, Uganda," British Journal of Cancer, vol. 92, no. 9, pp. 1808-1812, 2005.

[19] A. Gondos, E. Chokunonga, H. Brenner et al., "Cancer survival in a southern african urban population," International Journal of Cancer, vol. 112, no. 5, pp. 860-864, 2004.

[20] R. Sankaranarayanan, R. Swaminathan, H. Brenner et al., "Cancer survival in Africa, Asia, and Central America: a population-based study," The Lancet Oncology, vol. 11, no. 2, pp. $165-173$.

[21] H. M. A. Hamad, "Cancer initiatives in Sudan," Annals of Oncology, vol. 17, supplement 8, pp. viii32-viii36, 2006.

[22] H. G. Ahmed, A. S. Ali, and A. O. Almobarak, "Frequency of breast cancer among sudanese patients with breast palpable lumps," Indian Journal of Cancer, vol. 47, no. 1, pp. 23-26, 2010.

[23] A. E. Pinto, S. André, T. Pereira, S. Nóbrega, and J. Soares, "Prognostic comparative study of S-phase fraction and Ki-67 index in breast carcinoma," Journal of Clinical Pathology, vol. 54, no. 7, pp. 543-549, 2001.

[24] M. P. Mbonde, H. Amir, L. A. Akslen, and J. N. Kitinya, "Expression of oestrogen and progesterone receptors, Ki-67, p53 and BCL-2 proteins, cathepsin D, urokinase plasminogen activator and urokinase plasminogen activator-receptors in carcinomas of the female breast in an African population," East African Medical Journal, vol. 78, no. 7, pp. 360-365, 2001.

[25] H. Nalwoga, J. B. Arnes, H. Wabinga, and L. A. Akslen, "Frequency of the basal-like phenotype in African breast cancer," Acta pathologica, Microbiologica, et Immunologica Scandinavica, vol. 115, no. 12, pp. 1391-1399, 2007.

[26] K. D. Awadelkarim, C. Arizzi, E. O. Musa et al., "Basal-like phenotype in a breast carcinoma case series from Sudan: prevalence and clinical/pathological correlations," Pathology Research International, vol. 2011, Article ID 806831, 10 pages, 2011.

[27] P. Royston, D. G. Altman, and W. Sauerbrei, "Dichotomizing continuous predictors in multiple regression: a bad idea," Statistics in Medicine, vol. 25, no. 1, pp. 127-141, 2006. 


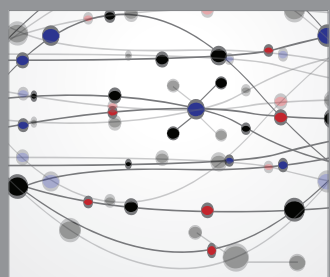

The Scientific World Journal
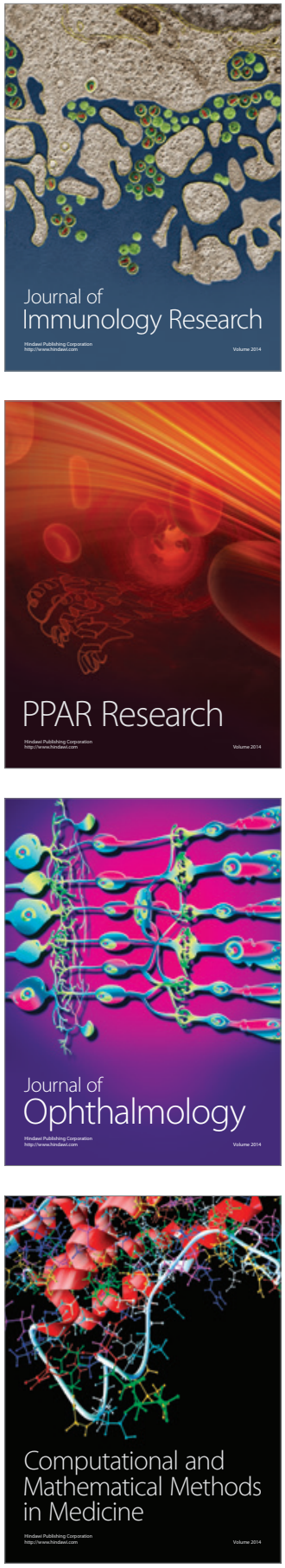

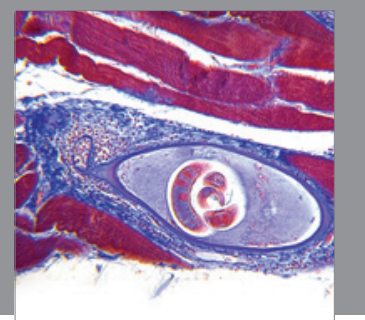

Gastroenterology

Research and Practice
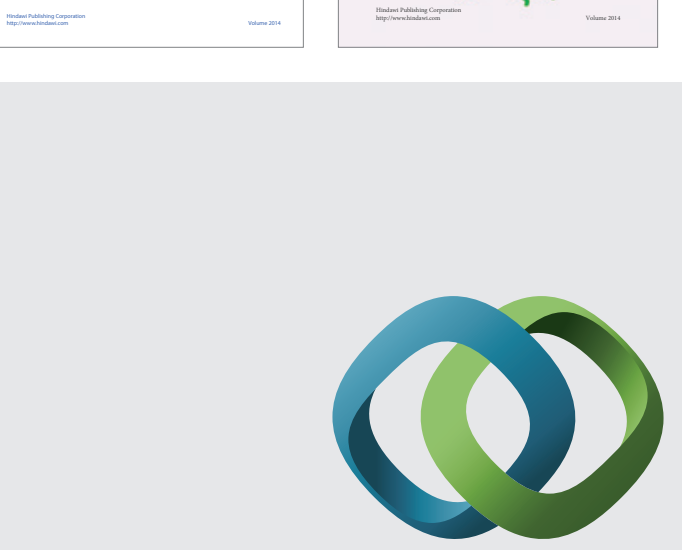

\section{Hindawi}

Submit your manuscripts at

http://www.hindawi.com
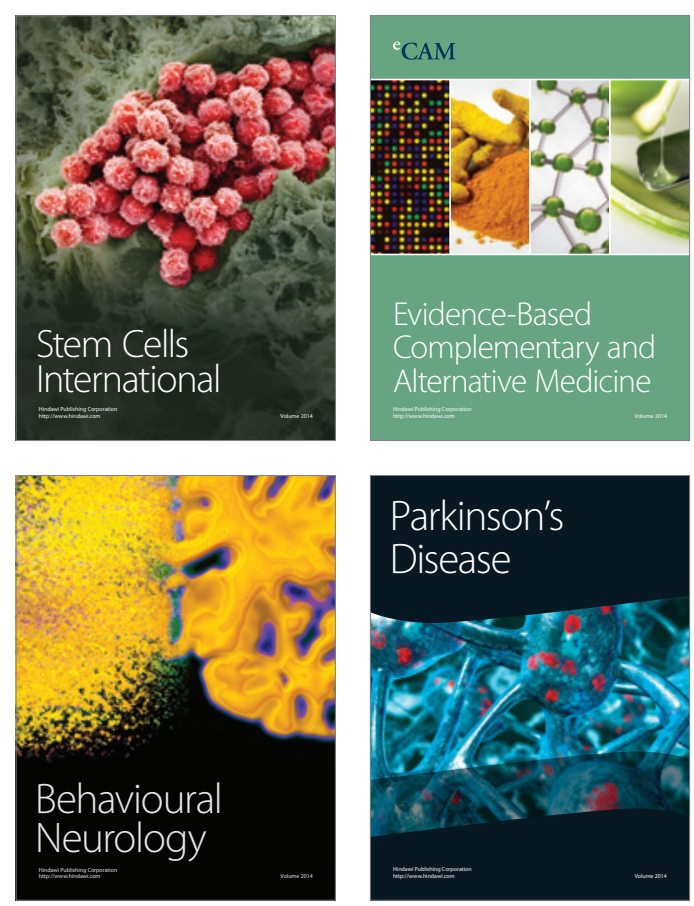

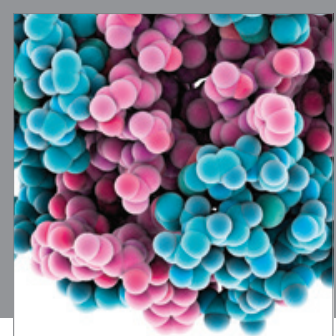

Journal of
Diabetes Research

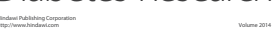

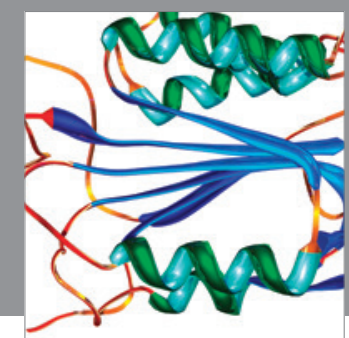

Disease Markers
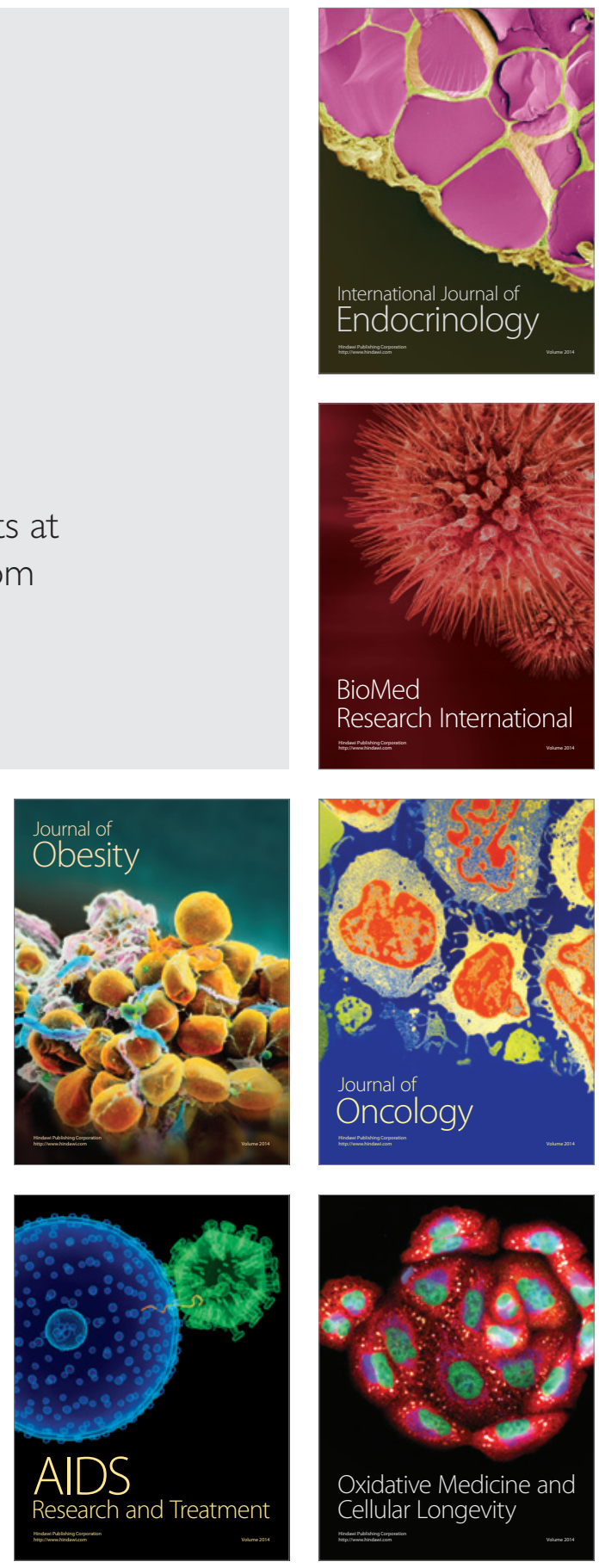